\title{
Familial Mediterranean Fever (FMF) with Proteinuria: Clinical Features, Histology, Predictors, and Prognosis in a Cohort of 25 Patients
}

\author{
Olga Kukuy, Avi Livneh, Aharon Ben-David, Juri Kopolovic, Alexander Volkov, Yael Shinar, \\ Eliezer Holtzman, Dganit Dinour, and Ilan Ben-Zvi
}

\begin{abstract}
Objective. Reactive (AA) amyloidosis may complicate familial Mediterranean fever (FMF), the prototype of autoinflammatory diseases. Thus, proteinuria in FMF is commonly viewed as resulting from amyloidosis, and kidney biopsy is deemed superfluous. However, nephropathy other than amyloidosis has been described in FMF, but its rate and distinctive characteristics are unknown. Our aim was to determine the rate and underlying pathology of FMF-related nonamyloidotic proteinuria and compare its clinical course, demographic, and genetic features to those of FMF-amyloid nephropathy.

Methods. This study is a retrospective analysis of data from patients with FMF undergoing kidney biopsy for proteinuria above $0.5 \mathrm{~g} / 24 \mathrm{~h}$, over 10 years (2001-2011). Clinical, laboratory, genetic, and pathology data were abstracted from patient files. Biopsies were viewed by an experienced pathologist, as necessary.

Results. Of the 25 patients referred for kidney biopsy, only 15 (60\%) were diagnosed with amyloid kidney disease (AKD), and 10 were diagnosed with another nephropathy. The AKD and nonamyloid kidney disease (NAKD) groups were comparable on most variables, but showed distinct characteristics with regard to the degree of proteinuria $(6.45 \pm 4.3 \mathrm{~g}$ vs $2.14 \pm 1.6 \mathrm{~g}, \mathrm{p}=0.006)$, rate of severe FMF (14 vs 5 patients, $\mathrm{p}=0.022$ ), and rate of development of end stage renal disease $(73.3 \%$ vs $20 \%, \mathrm{p}=0.015)$, respectively.

Conclusion. NAKD is common in FMF and, compared to amyloidosis, it is featured with milder course and better prognosis. Contrary to common practice, it is highly recommended to obtain a kidney biopsy from patients with FMF and proteinuria more than $0.5 \mathrm{~g} / 24 \mathrm{~h}$. (First Release Oct 15 2013; J Rheumatol 2013;40:2083-7; doi:10.3899/jrheum.130520)
\end{abstract}

Key Indexing Terms:

FAMILIAL MEDITERRANEAN FEVER PROTEINURIA AMYLOIDOSIS RENALFAILURE

\footnotetext{
From the Institute of Nephrology and Hypertension, Sheba Medical Center, Tel Hashomer; Heller Institute of Medical Research, Sheba Medical Center, Tel Hashomer; Department of Pathology, Sheba Medical Center, Tel Hashomer; Department of Pathology, Hadassah-Hebrew University Medical Center, Jerusalem; Sackler Faculty of Medicine, Tel Aviv University, Tel

Aviv; The Dr. Pinchas Borenstein Talpiot Medical Leadership Program 2012, Chaim Sheba Medical Center, Tel Hashomer, Israel.

Drs. Kukuy and Livneh contributed equally to this work.

O. Kukuy, MD, Institute of Nephrology and Hypertension, Sheba Medical Center; A. Livneh, MD, Heller Institute of Medical Research, Sheba Medical Center, Sackler Faculty of Medicine, Tel Aviv University; A. Ben-David, MD, Institute of Nephrology and Hypertension, Sheba Medical Center; J. Kopolovic, MD, Department of Pathology,

Hadassah-Hebrew University Medical Center; A. Volkov, MD, Department of Pathology, Sheba Medical Center; Y. Shinar, PhD, Heller Institute of Medical Research, Sheba Medical Center; E. Holtzman, MD, Institute of Nephrology and Hypertension, Sheba Medical Center and Sackler Faculty of Medicine, Tel Aviv University; D. Dinour, MD, Institute of Nephrology and Hypertension, Sheba Medical Center and Sackler Faculty of Medicine, Tel Aviv University; and I. Ben-Zvi, MD, Heller Institute of Medical Research, Sheba Medical Center, Sackler Faculty of Medicine, Tel Aviv University, and The Dr. Pinchas Borenstein Talpiot Medical Leadership Program 2012, Chaim Sheba Medical Center.

Address correspondence to Dr. Ilan Ben-Zvi. Department of Medicine F and The Zabludowicz Center for Autoimmune Diseases, Sheba Medical Center, Tel-Hashomer, 52621, Israel. Tel: +972544896620.

Fax: +9723530 8044. Email:Ilan.Benzvi@sheba.health.gov.il

Accepted August 8, 2013.
}

Familial Mediterranean fever (FMF) is a genetic, autoinflammatory disease prevailing in people living in the Mediterranean basin ${ }^{1}$. It is characterized by short spells (lasting several days) of febrile serositis, mediated by inflammatory cytokines ${ }^{2}$. The inflammation may continue in the clinically quiescent intervals, between the attacks, disposing patients to the development of reactive AA amyloidosis, the most severe complication of $\mathrm{FMF}^{3}$. In the majority of patients with FMF-amyloidosis, the kidneys are the first clinically affected organ. Their involvement with amyloidosis is manifested by proteinuria, progressing through phases of nephrotic and renal impairment to end stage kidney disease ${ }^{4,5}$. To date, colchicine is the only treatment that has been proven effective in the prevention of acute attacks and reactive amyloidosis in $\mathrm{FMF}^{6}$.

It is common practice to view proteinuria in FMF as resulting from amyloidosis and, therefore, avoid kidney biopsy in such a situation. This approach was based on observations made in large cohorts of FMF patients prior to the use of colchicine ${ }^{1,7}$. Nonetheless, kidney diseases, other than amyloidosis, that manifest with proteinuria and loss of

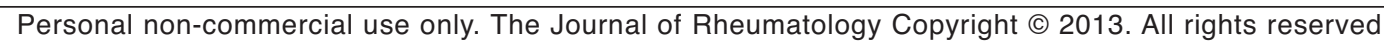


renal function have been described in $\mathrm{FMF}^{8}$. Following colchicine induced decrease in the rate of FMF-amyloidosis ${ }^{6,9}$, these nephropathies could become a major concern.

In spite of the overwhelming effectiveness of continuous colchicine prophylaxis, we still see up to 5 patients with FMF per year presenting with proteinuria exceeding 0.5 $\mathrm{g} / 24 \mathrm{~h}$ at our FMF center. Our study was undertaken to determine the current rate and pathological types of nonamyloidotic kidney disease (NAKD), presenting with proteinuria, in FMF. We also aimed to characterize the clinical, demographic, and genetic correlates of NAKD in an attempt to define possible traits, allowing for the prediction of the type of nephropathy, amyloid versus nonamyloid nephropathy, prior to kidney biopsy.

\section{MATERIALS AND METHODS}

The registry of the Israeli national center for FMF encompasses a patient population of about 12,000. Patient data are computerized and updated regularly at each patient followup visit (every 6 to 24 months), with mandatory pivotal clinical, laboratory, and treatment items, giving a clear picture of disease evolution along the course of the patient's disease.

This study is a retrospective analysis of observational data, collected continuously in a computerized system. We included in the study all FMF patients who underwent kidney biopsy for urine protein above $0.5 \mathrm{~g} / 24 \mathrm{~h}$ over the last 10 years (2001-2011). Approximately 10 patients who presented with proteinuria refused kidney biopsy.

All kidney biopsies were obtained and, when needed, reexamined by an experienced pathologist, specialized in kidney pathology. Kidney biopsies were studied using light, immunofluorescent, electron, and polarized microscopy, following appropriate sample preparation and staining (including Congo red). In the biopsy material from 3 patients, no glomeruli were left for immunofluorescence analysis. We also obtained the suspected prebiopsy diagnosis from the nephrologist directing the patient to kidney biopsy. Based on the pathological findings, the patients were divided into 2 groups: (A) amyloid kidney disease (AKD) group and (B) NAKD.

We abstracted data from patient files, including demographic, clinical, and genetic data, particularly focusing on the duration and severity of FMF, dose of colchicine, degree of adherence to colchicine treatment protocol during the course of the disease, evidence for comorbidities, rate of progression to end stage renal disease (ESRD), and cause of death. The severity of FMF was assessed by the Mor severity score ${ }^{10}$. The status of kidney disease at the time of kidney biopsy was assessed by the degree of proteinuria $(24 \mathrm{~h})$, presence of active urinary sediment $(>5 \mathrm{red}$ blood cells/high power field and/or cellular casts in microscopic urine examination), serum creatinine level, and blood pressure. Glomerular filtration rate was calculated using the modification of diet in renal disease or Cockcroft-Gault formulas and ESRD was defined as glomerular filtration rate $<15 \mathrm{ml} / \mathrm{min} / 1.73 \mathrm{~m}^{2}$. The presence of hypertension was defined by a systolic blood pressure above $140 \mathrm{~mm} \mathrm{Hg}$ and/or diastolic blood pressure above $90 \mathrm{~mm} \mathrm{Hg}$ on repetitive ambulatory measurements, or when patients were treated with antihypertensive agents. The onset of proteinuria was determined by the first urinalysis showing protein above $150 \mathrm{mg} / 24 \mathrm{~h}$.

For statistical analysis, the Mann-Whitney U test was used for comparing nonparametric variables between the AKD and NAKD groups. The Fisher exact test was performed for categorical measurements. All tests for significance were 2 tailed. Results were considered statistically significant if $p<0.05$. Directed logistic regression was performed to assess the impact of the factors that were found significant on the likelihood that patients would have AKD or NAKD.

The study was approved by the committee on human experimentation at the Sheba Medical Center, which waived the need to obtain informed consents, on the premise of retrospective analysis.

\section{RESULTS}

We reviewed 25 patients with FMF who underwent kidney biopsy for proteinuria exceeding $0.5 \mathrm{~g} / 24 \mathrm{~h}$. Of them, 15 patients (60\%) had AKD and $10(40 \%)$ had NAKD. Focal glomerulonephritis was the most common pathology in the NAKD group (Table 1). Before kidney biopsy, 6 of the 10 patients proven to suffer of NAKD were falsely diagnosed as having amyloidosis, while clinical, prebiopsy misdiagnosis in the 15 patients with AKD involved only 3 patients, who prior to biopsy were thought to have other kidney diseases.

The AKD and NAKD groups were comparable in their demographic and genetic characteristics (Table 2). Clinically, patients in the AKD group showed higher FMF severity score and at the time of kidney biopsy, had higher 24 hour protein excretion and higher rates of hypertension (Table 2). In contrast, at the same time, the rate of renal impairment as well as the prevalence of active urinary sediment were comparable in the 2 patient groups. Multivariate analysis found only hypertension to be more common in AKD than in NAKD, (OR 13.77, CI: $1.13-168.17, \mathrm{p}=0.04)$.

Evaluation of prognostic characteristics revealed that $73.3 \%$ of AKD group reached endstage renal disease during the study period, while in the other group the rate was only $20 \%, p=0.015$ (Table 3). Compared with the NAKD group, the AKD group showed a statistically insignificant trend to develop ESRD after a longer period of time from the onset of FMF attacks or of proteinuria. Similarly, the rate of death, which was higher in the AKD group, was not statistically significant. The causes of death are detailed in Table 3. They were variable, but progression of amyloidosis dominated.

\section{DISCUSSION}

In our study, we analyzed demographic, genetic, clinical, and histologic data of all FMF patients, who underwent kidney biopsy for proteinuria above $0.5 \mathrm{~g} / 24 \mathrm{~h}$, over a 10 year period. We found that $40 \%$ of our patient population displayed kidney pathology other than AA amyloidosis. This finding is unexpected and surprising, particularly in light of the accepted view that proteinuria in FMF is almost synonymous with the development of AA amyloidosis ${ }^{1,4,6}$,

Table 1. Spectrum of pathological findings in nonamyloid kidney disease (NAKD) group.

\begin{tabular}{lc}
\hline Kidney Disease & Number of Patients \\
\hline Focal glomerulonephritis & $5(50 \%)$ \\
Focal segmental glomerular sclerosis & $1(10 \%)$ \\
Immunoglobulin A nephropathy & $1(10 \%)$ \\
Minimal change disease & $1(10 \%)$ \\
Focal glomerulonephritis with features & \\
$\quad$ of thrombotic microangiopathy & $1(10 \%)$ \\
Focal interstitial nephritis & $1(10 \%)$ \\
\hline
\end{tabular}


Table 2. Demographic, clinical, and genetic data from the 2 study groups.

\begin{tabular}{lccc}
\hline Parameters & $\begin{array}{c}\text { Amyloid Kidney } \\
\text { Disease (AKD) } \\
(\mathrm{n}=15)\end{array}$ & $\begin{array}{c}\text { Nonamyloid Kidney } \\
\text { Disease (NAKD) } \\
(\mathrm{n}=10)\end{array}$ & $\mathrm{p}$ \\
\hline Age on biopsy, yrs & $42.8 \pm 14.4$ & $41.6 \pm 12.8$ & 0.952 \\
Male sex, $\mathrm{n}$ & 8 & 8 & 0.229 \\
North African origin, $\mathrm{n}$ & 13 & 6 & 0.175 \\
Severe FMF, $\mathrm{n}$ & 14 & 5 & 0.022 \\
Mean creatinine level at time of biopsy, mg/dl & $1.53 \pm 0.9$ & $1.31 \pm 0.4$ & 0.825 \\
Degree of proteinuria at biopsy, g/24 h & $6.45 \pm 4.3$ & $2.14 \pm 1.6$ & 0.006 \\
Proteinuria $\geq 3.5$ g/24 h, nephrotic range & 11 & 2 & 0.015 \\
Hypertension, $\mathrm{n}$ & 10 & 1 & 0.011 \\
Active urine sediment, $\mathrm{n}$ & 13 & 6 & 0.357 \\
Homozygosity for M694V pyrin mutation, $\mathrm{n}$ & 13 & $3-\mathrm{M} 694 \mathrm{~V} / 0$ & 0.175 \\
Non M694V homozygous genotypes & $1-\mathrm{M} 694 \mathrm{~V} / 0$ & $\mathrm{~N} / \mathrm{A}$ \\
& 1-M694V/E148Q & 1-M694V/V726A & \\
\hline
\end{tabular}

FMF: familial Mediterranean fever; N/A: not applicable.

Table 3. Prognostic characteristics of the study population.

\begin{tabular}{|c|c|c|c|}
\hline Variables & $\begin{array}{l}\text { Amyloid Kidney Disease (AKD) } \\
\qquad(\mathrm{n}=15)\end{array}$ & $\begin{array}{l}\text { Nonamyloidotic Kidney Disease (NAKD) } \\
\qquad(\mathrm{n}=10)\end{array}$ & $\mathrm{p}$ \\
\hline ESRD/transplant at the study end, $\%$ & 73.3 & 20 & 0.015 \\
\hline Time interval from onset of FMF until ESRD, yrs & $41 \pm 13.6$ & $23.5 \pm 2.9$ & 0.202 \\
\hline Death, $\%$ & 40 & 10 & 0.179 \\
\hline Causes of death & $\begin{array}{l}\text { Progression of amyloidosis, } \mathrm{n}=3 \\
\text { Septic foot, } \mathrm{n}=1 \\
\text { Creutzfeldt-Jacob disease, } \mathrm{n}=1 \\
\text { Unknown, } \mathrm{n}=1\end{array}$ & Uncontrolled bleeding and pneumonia, $\mathrm{n}=1$ & \\
\hline
\end{tabular}

ESRD: end stage renal disease; FMF: familial Mediterranean fever.

and because of the common practice to increase colchicine, the only available agent for FMF amyloidosis, to its maximal tolerated dose in all cases of FMF manifested with proteinuria. To the best of our knowledge, the only published work on this subject is by Said, et $a l^{8}$, who found that around 50\% of FMF nephropathy is caused by NAKD, comparable to our results.

We could not establish any clinical or laboratory measure as a surrogate marker for kidney biopsy, capable of diagnosing amyloidosis in patients with FMF presenting with proteinuria. Indeed, the finding of amyloidosis on biopsy was significantly associated with more severe FMF, the presence of hypertension, and higher levels of proteinuria at the time of biopsy, but these findings are not helpful for the individual patient and the level of proteinuria depends on its duration, which was not normalized for patient cohorts in our study. We could not exploit even the traditional evolution of AA amyloidosis kidney disease (slow advancement to nephrotic range proteinuria and subsequent decline of kidney function to ESRD) ${ }^{4,5}$, to distinguish between AKD and NAKD, as some patients with amyloidosis presented with renal impairment concomitantly with proteinuria, without going through a nephrotic phase. Finally, urinalysis was not discerning either and contained active sediment in the 2 groups, contrasting our view that amyloidotic patients usually present with relatively pure proteinuria.

Altogether, the above findings support performing renal biopsy to obtain a correct diagnosis in all patients with FMF presenting with significant proteinuria. Clinical prediction is not adequate, as prebiopsy evaluation failed to establish a correct diagnosis in $36 \%$ of the study population and in $60 \%$ of the patients with NAKD. For comparison, prebiopsy diagnosis of subtypes of lupus nephritis is much more accurate $^{11}$. Nevertheless, kidney biopsy in lupus nephritis is considered obligatory, even though its contribution to treatment decision above clinical measures is questionable ${ }^{12,13}$.

The importance of kidney biopsy stems from the resulting selection of therapeutic approach: raising colchicine to the maximal tolerable dose, which at times might be dangerous, versus other treatments, including steroids or other immunosuppressive agents and biologic

Personal non-commercial use only. The Journal of Rheumatology Copyright @ 2013. All rights reserved. 
medications. Also, biopsy results may affect whether patients adhere to the advised treatment (only a quarter of our patients in the 2 groups were compliant with colchicine). Applying new anti-AA amyloidosis medications, including anti-interleukin (IL) 1, anti-IL-6 anti-tumor necrosis factor, or kiacta ${ }^{14,15,16,17}$, if approved, also requires biopsy. It is questionable whether glomerulopathies other than amyloidosis are also responsive to colchicine dose increment ${ }^{18,19}$.

Kidney biopsy is important for prognostic considerations. Many more patients in the AKD group reached ESRD and, although not statistically significant, more patients died, mostly from the progression of the disease. Patients should be aware of their fate and chances of receiving kidney replacement therapy, which was more common in the AKD group (Table 3). The longer time from the onset of FMF or proteinuria to ESRD in the AKD group reflects the indolent nature of kidney disease in AA amyloidosis, as noted above ${ }^{4,5}$. Yet, AA amyloidosis is a systemic disease, which involves many other organs, and therefore eventually runs a more severe course with worse outcome, compared to NAKD, as appears here, with higher rates of death and ESRD.

The rate of hypertension was significantly higher in the AKD group than in the NAKD group, despite comparable kidney function. Moreover, the multivariate analysis makes it the only factor that could discriminate between the 2 groups. One possible explanation for this finding is arterial stiffness, secondary to chronic inflammation, underlying AA amyloidosis $20,21,22$. However, the rate of hypertension in AA amyloidosis is controversial. Tuglular, et al found hypertension in only $12 \%$ of 287 patients with secondary amyloidosis (64\% due to FMF) ${ }^{23}$. Compared to Tuglular, et $a l^{23}$, Hazenberg and Rijswijk ${ }^{24}$ noted a higher rate of hypertension (20\% to $35 \%)$ in their study on AA amyloidosis. In another article, 8 of 9 female patients with rheumatoid arthritis and AA amyloidosis had hypertension ${ }^{25}$. It might be that the conflicting results arise, in part, from misdiagnosing NAKD as AKD, based on clinical grounds alone. Whether hypertension had a role in the deleterious outcome of AKD in this study is unknown, but it may serve as a target for treatment in future patients with AKD.

Various types of glomerulonephritides have been described in patients with $\mathrm{FMF}^{26}$. In Said, et al, the nonamyloid cohort consisted mainly of IgA nephropathy ${ }^{8}$. It is possible that in our study, some patients diagnosed with focal glomerulonephritis actually had undiagnosed IgA nephropathy, as 3 biopsies from the NAKD group were not studied with anti-IgA antibodies due to lack of glomeruli. It is still unclear whether the abundance of $\operatorname{IgA}$ nephropathy in Said, et $a l^{8}$ is due merely to a coincidence, in accord with the notion that $\operatorname{IgA}$ nephropathy is the most common glomerulopathy worldwide ${ }^{27}$, or due to a preferential expression in FMF, similar to some other inflammatory conditions prevailing in FMF, such as inflammatory bowel disease, Behçet disease, Henoch-Schönlein purpura, and polyarteritis nodosa ${ }^{28,29,30,31}$.

The retrospective character of our study limits insight into the natural history of the disease, including: (A) Accurate temporal relations between the main features of kidney disease (proteinuria, active urinary sediment, and renal malfunction). (B) The association of these features with FMF activity and colchicine dose in both groups. (C) The role of hypertension in the development of proteinuria and loss of renal function in FMF-related kidney disease. Nevertheless, our study was carried out in one of the largest FMF populations available worldwide, where ongoing followup of patients' clinical and nephrologic measures is performed periodically, allowing for us to safely analyze the variables evaluated in our study.

Among patients with FMF and evolving proteinuria, we have identified a cohort with kidney pathology different from AA amyloidosis. Compared with AA amyloidosis, this group was characterized by a milder FMF phenotype, non-nephrotic range proteinuria, normal blood pressure, and a less aggressive course of kidney disease. Consequently, significantly less NAKD patients reached ESRD at the end of the followup period and their survival rate was higher. Thus, the accurate diagnosis of renal pathology in FMF patients with proteinuria should be established by kidney biopsy, since it is clinically unpredictable and has important treatment and prognostic implications.

\section{REFERENCES}

1. Sohar E, Gafni J, Pras M, Heller H. Familial Mediterranean fever. A survey of 470 cases and review of the literature. Am J Med 1967;43:227-53.

2. Ben-Zvi I, Livneh A. Chronic inflammation in FMF: markers, risk factors, outcomes and therapy. Nat Rev Rheumatol 2011;7:105-12.

3. Yalçinkaya F, Cakar N, Acar B, Tutar E, Güriz H, Elhan AH, et al. The value of the levels of acute phase reactants for the prediction of familial Mediterranean fever associated amyloidosis: a case control study. Rheumatol Int 2007;27:17-22.

4. Heller H, Sohar E, Gafni J, Heller J. Amyloidosis in familial Mediterranean fever. Arch Intern Med 1961;107:539-50.

5. Zemer D, Livneh A, Pras M, Sohar E. The kidney in familial Mediterranean fever. Contribute Nephrology 1993;102:187-97.

6. Zemer D, Pras M, Sohar E, Modan M, Cabili S, Gafni J. Colchicine in the prevention and treatment of the amyloidosis of familial Mediterranean fever. N Engl J Med 1986;314:1001-5.

7. Gafni J, Ravid M, Sohar E. The role of amyloidosis in familial Mediterranean fever. A population study. Isr J Med Sci 1968; 4:995-9.

8. Said R, Hamzeh Y, Said S, Tarawneh M, al-Khateeb M. Spectrum of renal involvement in familial Mediterranean fever. Kidney Int 1992;41:414-9.

9. Livneh A, Zemer D, Langevitz P, Laor A, Sohar E, Pras M. Colchicine treatment of AA amyloidosis of familial Mediterranean fever. An analysis of factors affecting outcome. Arthritis Rheum 1994:37:1804-11.

10. Mor A, Shinar Y, Zaks N, Langevitz P, Chetrit A, Shtrasburg S, et al. Evaluation of disease severity in familial Mediterranean fever. Semin Arthritis Rheum 2005;35:57-64.

11. Esdaile JM, Levinton C, Federgreen W, Hayslett JP, Kashgarian M.

Personal non-commercial use only. The Journal of Rheumatology Copyright @ 2013. All rights reserved. 
The clinical and renal biopsy predictors of long-term outcome in lupus nephritis: a study of 87 patients and review of the literature. Q J Med 1989;72:779-833.

12. Mittal B, Rennke H, Singh AK. The role of kidney biopsy in the management of lupus nephritis. Curr Opin Nephrol Hypertens 2005;14:1-8.

13. Bomback AS, Appel GB. Updates on the treatment of lupus nephritis. J Am Soc Nephrol 2010;21:2028-35.

14. Stankovic Stojanovic K, Delmas Y, Torres PU, Peltier J, Pelle G, Jéru I, et al. Dramatic beneficial effect of interleukin-1 inhibitor treatment in patients with familial Mediterranean fever complicated with amyloidosis and renal failure. Nephrol Dial Transplant 2012;27:1898-901.

15. Hakala M, Immonen K, Korpela M, Vasala M, Kauppi MJ. Good medium-term efficacy of tocilizumab in DMARD and anti-TNF- $\alpha$ therapy resistant reactive amyloidosis. Ann Rheum Dis 2013;72:464-5.

16. Nakamura T, Higashi S, Tomoda K, Tsukano M, Baba S. Efficacy of etanercept in patients with AA amyloidosis secondary to rheumatoid arthritis. Clin Exp Rheumatol 2007;25:518-22.

17. Rumjon A, Coats T, Javaid MM. Review of eprodisate for the treatment of renal disease in AA amyloidosis. Int J Nephrol Renovasc Dis 2012;5:37-43.

18. Koukoui L, Blau A, Kopolovic J, Pras M, Livneh A. A possible favorable effect of colchicine in IgA nephropathy in a carrier of a MEFV mutation. Clin Nephrol 2004;62:226-8.

19. Rigante D, Federico G, Ferrara P, Maggiano N, Avallone L, Pugliese AL, et al. IgA nephropathy in an Italian child with familial Mediterranean fever. Pediatr Nephrol 2005;20:1642-4.

20. Mahmud A, Feely J. Arterial stiffness is related to systemic inflammation in essential hypertension. Hypertension 2005;46:1118-22.

21. Pietri P, Vyssoulis G, Vlachopoulos C, Zervoudaki A, Gialernios T, Aznaouridis K, et al. Relationship between low-grade inflammation and arterial stiffness in patients with essential hypertension. J Hypertens 2006;24:2231-8.
22. Yildiz M, Masatlioglu S, Seymen P, Aytac E, Sahin B, Seymen HO. The carotid-femoral (aortic) pulse wave velocity as a marker of arterial stiffness in familial Mediterranean fever. Can J Cardiol 2006;22:1127-31.

23. Tuglular S, Yalcinkaya F, Paydas S, Oner A, Utas C, Bozfakioglu S, et al. A retrospective analysis for aetiology and clinical findings of 287 secondary amyloidosis cases in Turkey. Nephrol Dial Transplant 2002;17:2003-5.

24. Hazenberg BP, van Rijswijk MH. Clinical and therapeutic aspects of AA amyloidosis. Baillieres Clin Rheumatol 1994;8:661-90.

25. Ueno T, Takeda K, Nagata M. Remission of proteinuria and preservation of renal function in patients with renal AA amyloidosis secondary to rheumatoid arthritis. Nephrol Dial Transplant 2012;27:633-9.

26. Akpolat T, Akpolat I, Karagoz F, Yilmaz E, Kandemir B, Ozen S. Familial Mediterranean fever and glomerulonephritis and review of the literature. Rheumatol Int 2004;24:43-5.

27. Galla JH. IgA nephropathy. Kidney Int 1995;47:377-87.

28. Fidder HH, Chowers Y, Lidar M, Sternberg M, Langevitz P, Livneh A. Crohn disease in patients with familial Mediterranean fever. Medicine 2002;81:411-6.

29. Rabinovich E, Livneh A, Langevitz P, Brezniak N, Shinar E, Pras $\mathrm{M}$, et al. Severe disease in patients with rheumatoid arthritis carrying a mutation in the Mediterranean fever gene. Ann Rheum Dis 2005;64:1009-14.

30. Schwartz T, Langevitz P, Zemer D, Gazit E, Pras M, Livneh A. Behçet's disease in Familial Mediterranean fever: characterization of the association between the two diseases. Semin Arthritis Rheum 2000;29:286-95.

31. Ozen S, Ben-Chetrit E, Bakkaloglu A, Gur H, Tinaztepe K, Calguneri M, et al. Polyarteritis nodosa in patients with Familial Mediterranean Fever (FMF): a concomitant disease or a feature of FMF? Semin Arthritis Rheum 2001;30:281-7. 